\title{
Neprilysin Gene Transfer Reduces Human Amyloid Pathology in Transgenic Mice
}

\author{
Robert A. Marr, ${ }^{1}$ Edward Rockenstein, ${ }^{2}$ Atish Mukherjee, ${ }^{4}$ Mark S. Kindy, ${ }^{5}$ Louis B. Hersh, ${ }^{4}$ Fred H. Gage, ${ }^{1}$ \\ Inder M. Verma, ${ }^{1}$ and Eliezer Masliah ${ }^{2,3}$ \\ ${ }^{1}$ Laboratory of Genetics, The Salk Institute for Biological Studies, La Jolla, California 92037, Departments of ${ }^{2}$ Neurosciences and ${ }^{3}$ Pathology, The University \\ of California San Diego, La Jolla, California 92093, and ${ }^{4}$ Department of Biochemistry, The University of Kentucky, Lexington, Kentucky 40536, and \\ ${ }^{5}$ Department of Physiology and Neuroscience, Medical University of South Carolina, Charleston, SC 29425
}

The degenerative process of Alzheimer's disease is linked to a shift in the balance between amyloid- $\beta$ (A $\beta$ ) production, clearance, and degradation. Neprilysin has recently been implicated as a major extracellular A $\beta$ degrading enzyme in the brain. However, there has been no direct demonstration that neprilysin antagonizes the deposition of amyloid- $\beta$ in vivo. To address this issue, a lentiviral vector expressing human neprilysin (Lenti-Nep) was tested in transgenic mouse models of amyloidosis. We show that unilateral intracerebral injection of Lenti-Nep reduced amyloid- $\beta$ deposits by half relative to the untreated side. Furthermore, Lenti-Nep ameliorated neurodegenerative alterations in the frontal cortex and hippocampus of these transgenic mice. These data further support a role for neprilysin in regulating cerebral amyloid deposition and suggest that gene transfer approaches might have potential for the development of alternative therapies for Alzheimer's disease.

Key words: neprilysin; Alzheimer's disease; lentivirus; gene therapy; amyloid- $\beta$; endopeptidase

\section{Introduction}

Abnormal production and accumulation in the CNS of amyloid- $\beta$ (A $\beta)$ protein, a proteolytic product of amyloidprecursor-protein (APP) metabolism, is currently being investigated as one of the central mechanisms involved in the pathogenesis of Alzheimer's disease (AD). Although in familial forms of AD mutations in the APP and presenilin (PS) genes result in increased production of $\mathrm{A} \beta_{1-42}$, in sporadic forms the mechanism is less clear. Some studies point to decreased $A \beta_{1-42}$ clearance, and others suggest that a shift in the balance between $\mathrm{A} \beta$ production and degradation might be responsible for the disease (Glabe, 2000).

Neprilysin (Nep) has been recently identified as a major extracellular $\mathrm{A} \beta$ degrading enzyme in the brain (Iwata et al., 2000). Neprilysin (neutral endopeptidase, EC 3.4.24.11, enkephalinase, $\mathrm{CD} 10)$ is a $97 \mathrm{kDa}$ cell surface-associated zinc metalloendopeptidase that functions to degrade peptide signaling molecules in the circulatory, immune, and nervous systems (Turner et al.,

\footnotetext{
Received 0ct. 23, 2002; revised Dec. 4, 2002; accepted Dec. 27, 2002

R.A.M. was supported in part by funds from the Canadian Institutes of Health Research. M.S.K. is supported by Grant AG 19323 from National Institute on Aging. This work was supported in part by funds from the Alzheimer's Association (L.B.H.). F.H.G. is supported by National Institutes of Health Grant AG08514, the Lookout Fund, the Fox Foundation, and the National Parkinson Foundation. I.M.V. is an American Cancer Society Professor of Molecular Biology and is supported by grants from National Institutes of Health, the Wayne and Gladys Valley Foundation, and the H. N. and Frances C. Berger Foundation. E.M. is supported by National Institutes of Health Grants AG5131, AG10689, and AG18440 and by a grant from the M. J. Fox Foundation for Parkinson's Research. We thank Marylyn Gage for critical reading of this manuscript.

Correspondence should be addressed to either of the following: Inder M. Verma, Laboratory of Genetics, The Salk Institute for Biological Studies, 10010 North Torrey Pines Road, La Jolla, California 92037, E-mail: verma@salk.edu; or Fred H. Gage, Laboratory of Genetics, The Salk Institute for Biological Studies, 10010 North Torrey Pines Road, La Jolla, California 92037, E-mail: gage@salk.edu.

Copyright @ 2003 Society for Neuroscience $\quad 0270-6474 / 03 / 231992-05 \$ 15.00 / 0$
}

2001). Recent studies have shown that neprilysin knock-out mice exhibit a gene dose-dependent increase in $\mathrm{A} \beta$ levels in the brain (Iwata et al., 2001) that is comparable with that associated with presenilin mutations. Additionally, Nep is reduced in areas vulnerable to plaque formation (Akiyama et al., 2001; Reilly, 2001; Yasojima et al., 2001a,b), and downregulation of neprilysin with chemical inhibitors results in increased $\mathrm{A} \beta$ concentrations in the brain (Iwata et al., 2000). Infection of primary neurons with a Sindbis vector expressing neprilysin also reduces $\mathrm{A} \beta$ production in vitro (Hama et al., 2001). Taken together, these studies suggest that neprilysin plays a key role in the clearance of $A \beta$. To further investigate the role of neprilysin in amyloid deposition, a lentiviral vector expressing human neprilysin (Lenti-Nep) was prepared and tested in transgenic models of amyloidosis. This approach was favored over other viral vectors because lentiviral vectors have been shown to efficiently transduce cells of the CNS, and they are not cytotoxic (Naldini et al., 1996; Dull et al., 1998; Miyoshi et al., 1998). Furthermore, expression of the therapeutic product can theoretically be sustained for the life span of the animal.

\section{Materials and Methods}

Cell culture and medium. 293T cells were cultured in DMEM (Cellgro, Kansas City, MO) + 10\% FBS (HyClone, Logan, UT) + antibiotic/ antimycotic (Life Technologies, Grand Island, NY). The rat hippocampal neural progenitor (HCN) cell line (Ray et al., 1995) was cultured in DMEM/Hams/F-12 (high glucose) (Omega Scientific, Tarzana, CA) supplemented with $1 \mathrm{~mm}$ L-glutamine, (Sigma, St. Louis, MO), $1 \times$ penicillin/streptomycin/fungizone (Life Technologies), $1 \times \mathrm{N} 2$ supplement (Life Technologies), and $20 \mathrm{ng} / \mathrm{ml}$ basic fibroblast growth factor (bFGF) (Life Technologies) on plates coated with poly-L-ornithine (Sigma) and laminin (Fisher, Houston, TX). For differentiation, HCN cells were 
grown in the above medium without bFGF, and in the presence of $0.5 \%$ fetal bovine serum and $1 \mu \mathrm{M}$ retinoic acid (Sigma).

Lentiviral vector production. Vector plasmids were constructed for the production of third generation lentiviral vectors that expressed the neprilysin gene. The human cytomegalovirus (CMV) promoter was used to drive expression of the transgenes (see Fig. 1a). Vector plasmids were also constructed expressing green fluorescent protein (GFP) and a point mutant of neprilysin (E585V) (Hama et al., 2001). Additional control experiments were performed by heat inactivating $\left(70^{\circ} \mathrm{C}\right.$ for $\left.20 \mathrm{~min}\right)$ the vector (Lenti-iNep). All vectors were designed to be self-inactivating (Miyoshi et al., 1998) and used the woodchuck hepatitis virus post-transcriptional regulatory element (WPRE) 3' to the transgene (Zufferey et al., 1999). The HIV-1 central poly-purine track was also located $5^{\prime}$ to the promoter (Follenzi et al., 2000). We produced lentiviral vectors using a fourplasmid transfection system, as described previously (Dull et al., 1998; Miyoshi et al., 1998). Briefly, 293T cells were transfected with vector and packaging plasmids, the supernatants were collected, and vectors were concentrated by centrifugation. The lentiviral vector titers were estimated by measuring the amount of HIV p24 gag antigen with an ELISA kit (Perkin-Elmer Life Science, Boston, MA) [100,000 transducing units (TU) per nanogram of p24] or by flow cytometry (below).

Flow cytometry. 293T cells were plated into 24-well plates (coated with poly-L-lysine) (Sigma) and counted before infection. Infections were done by making serial dilutions of the lentiviral vector preparation in culture medium and incubating overnight in a volume of $200 \mu \mathrm{l}$. The next day the transduced cells were washed with fresh medium and incubated for 3-4 d. The transduced cells were then removed with an EDTA buffer and washed in PBS. For Lenti-Nep and Lenti-NepX (Fig. 1a), the cell pellet was then resuspended in $50 \mu \mathrm{l}$ of PBS/antibody solution containing $10 \mu \mathrm{l}$ of FITC-conjugated anti-CD10 antibody (CD10F) (Research Diagnostics, Flanders, NJ) and incubated for $1 \mathrm{hr}$ at $4^{\circ} \mathrm{C}$. Afterward the cells were washed twice in PBS and analyzed by flow cytometry. For Lenti-GFP, transduced cells were simply collected (as above) and directly analyzed by flow cytometry. The percentage of cells positive was used to determine the number of transducing units per unit volume.

Transgenic mouse generation and intracerebral injections of lentiviral vectors. For this study two independent lines of human (h) APP tg mice were used. The first expressed mutant human amyloid precursor protein cDNA (hAPP ${ }^{\mathrm{V} 717 \mathrm{~F}}$ ) under the control of the platelet-derived growth factor promoter (PDAPP minigene) (Masliah et al., 1996). The second expressed double-mutant hAPP London (V717I) and Swedish (K670M/ N671L) under the control of the murine Thyl regulatory sequences (Rockenstein et al., 2001). A total of 13 PD-hAPP tg mice from line J9M, aged 12-20 months, and 13 mThyl-hAPP from line 41 (TASD41), aged 11-14 months, were injected with $3 \mu \mathrm{l}$ of the lentiviral preparations $\left(1.5 \times 10^{7} \mathrm{TU}\right)$ into the frontal cortex and hippocampus (using a $5 \mu \mathrm{l}$ Hamilton syringe, $0.25 \mu \mathrm{l} / \mathrm{min}$ ). Mice received unilateral injections (right side) to allow comparisons against the contralateral side, with Lenti-Nep, Lenti-NepX (inactive), Lenti-iNep (heat inactivated), LentiGFP, or vehicle alone. One month after injection mice were killed, and the brains were immersion-fixed in $4 \%$ paraformaldehyde for subsequent immunohistochemical analysis.

Immunoblots. For the detection of neprilysin in vitro, transduced cells (1.0 ng of p24 per cell) were lysed in RIPA lysis buffer plus protease inhibitors (Complete mini) (Roche, Indianapolis, IN). The lysates were analyzed by SDS-PAGE on a 7\% Tris-acetate polyacrylamide gel $(\mathrm{Nu}-$ PAGE) (Invitrogen, Carlsbad, CA). Immunoblots were performed with a primary anti-CD10 antibody (clone 56C6, 1:75 dilution, overnight) (Research Diagnostics) and a secondary goat anti-mouse-IgG-HRP antibody (1:5000, 2 hr) (Santa Cruz Biotechnology, Santa Cruz, CA) and visualized by chemiluminescence (Amersham Biosciences, Baied d'Urfe, Canada).

For the detection of neprilysin expressed in vivo, $3 \mu \mathrm{l}$ of Lenti-Nep $\left(1.5 \times 10^{7} \mathrm{TU}\right)$ was injected directly into the hippocampus (using a stereotaxic frame). For negative controls, mice were injected with LentiGFP at the same concentration or with saline. One week later the brains were removed, and the right hemispheres were homogenized in RIPA buffer plus protease inhibitors (Complete mini) (Roche) on ice. Protein concentrations were determined by the Bradford method (Bio-Rad, Her- cules, CA). The homogenates were immunoprecipitated overnight $\left(4^{\circ} \mathrm{C}\right)$ with $20 \mu$ preswollen protein $\mathrm{G}$ Sepharose (Amersham Biosciences) and $1 \mu \mathrm{l}$ of anti-CD10 antibody (CLONE SN5c/L4-1A1, $1 \mathrm{mg} / \mathrm{ml}$ ) (Ancell, Bayport, MN). After washing, precipitated proteins were extracted in protein loading buffer and analyzed by immunoblot as described above.

Neprilysin activity assay. Neprilysin activity was measured as described previously (Li and Hersh, 1995). Briefly, whole cell or tissue homogenates (293T or human kidney) were measured using glutaryl-Ala-AlaPhe-4-methoxy-2-naphthylamide as a substrate. Protein was quantified using the BCA reaction. The addition of phosphoramide (a neprilysin inhibitor) caused a $95 \%$ reduction in activity.

Amyloid- $\beta$ degradation assays. Lentiviral vectors were used to transduce $293 \mathrm{~T}$ or HCN cells in a 24-well plate. Two days after transduction the medium was removed, and the cells were washed and incubated with medium spiked with $\mathrm{A} \beta_{1-42}$ or $\mathrm{A} \beta_{1-40}(1000 \mathrm{pg} / \mathrm{ml}$; Biosource International, Camarillo, CA) $+1 \mu \mathrm{MnCl}_{2}$ (Sigma) (without supplements or serum). The medium was removed $6-8 \mathrm{hr}$ later and gently centrifuged to avoid cell contamination. The medium was then assayed for $\mathrm{A} \beta_{1-42}$ or

a
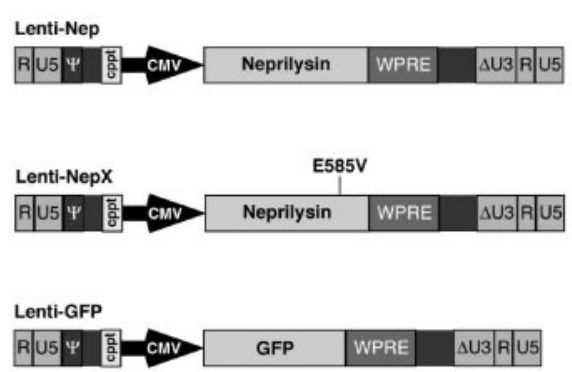

b
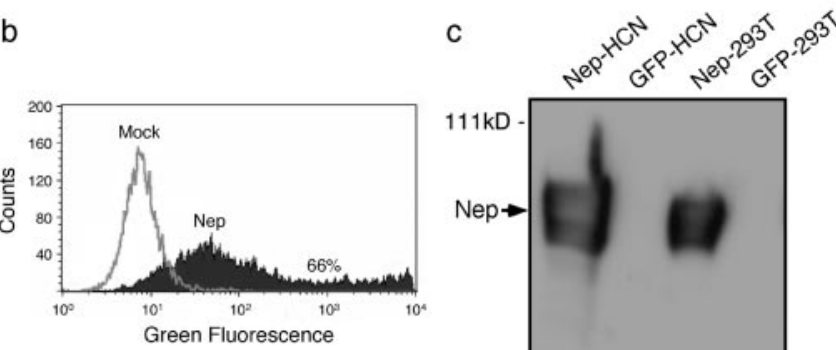

d

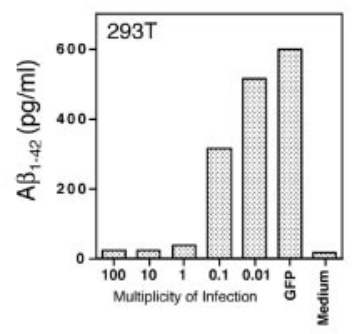

e

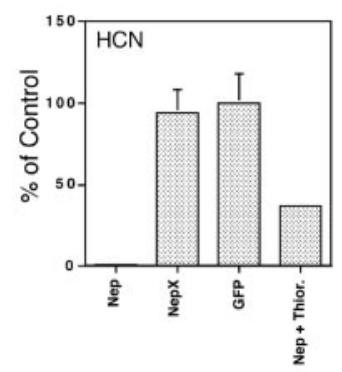

Figure 1. a, Vector design showing the neprilysin, inactive neprilysin (NepX), and green fluorescent protein (GFP) expressing lentiviral constructs. The internal promoters driving the transgenes are indicated by arrows (human CMV). LTR sequences are shown on the ends (packaging signal, $\Psi$ ). b, 293T cells transduced with Lenti-Nep (10 nl per well, or 0.015 pg p24 gag-antigen per (ell) were immunostained for neprilysin expression and analyzed by flow cytometry. c, Immunoblot on 293T cells and differentiated rat neural progenitors (HCN) transduced with the Lenti-Nep or Lenti-GFP vector. d, 293T cells transduced with serial dilutions of Lenti-Nep could degrade $A \beta_{1-42}$ in a dose-dependent manner. Lenti-GFP transduced cells were used as a negative control (GFP), whereas medium alone was used to determine the baseline (Medium). e, Differentiated neural progenitors (HCN) infected with Lenti-Nep (Nep; $n=2)$, Lenti-NepX (NepX; $n=2)$ or Lenti-GFP $(G F P ; n=2)$ were used to degrade $A \beta_{1-42}$ (as above). Values are presented as the percentage of the average value from the GFP samples. Thiorphan was also used as a negative control (Nep + Thior.; $n=1)$. 
$\mathrm{A} \beta_{1-40}$ using isoform-specific ELISA kits (Biosource International). Cells infected with Lenti-GFP and Lenti-NepX (E585V) were used as negative controls. Medium plus $\mathrm{ZnCl}_{2}$ was used to determine background signal for the ELISA assays. Thiorphan (Sigma) was also used as a negative control $(150 \mu \mathrm{M})$.

Immunohistochemical and neuropathological analyses. The fixed brains were serially sectioned at $40 \mu \mathrm{m}$ with a Vibratome 2000 (Leica, New York, NY) and sections were immunolabeled as described previously with antibodies against A $\beta$ (3D6) (Elan Pharmaceutical, South San Francisco, CA), MAP2 (Chemicon, Pittsburgh, PA), or neprilysin (56C6) (Research Diagnostics) followed by incubation with FITC-conjugated secondary antibodies and were imaged with the laser scanning confocal microscope (LSCM) (Mucke et al., 2000). The percentage of the area of the neuropil occupied by $\mathrm{A} \beta$-immunoreactive (IR) plaques was used to estimate amyloid load (10 random fields from three sections of the hippocampus), whereas the percentage of the area of the neuropil occupied by MAP2-IR dendrites was used to evaluate the damage or preservation of the neuronal structure. Additional double-immunolabeling experiments were performed to ascertain colocalization of lentiviral neprilysin expression and amyloid plaques. For this purpose, vibratome sections were incubated with antibodies against $\mathrm{A} \beta$ (3D6) and Nep (56C6) (Research Diagnostics) and visualized with Tyramide Red (for Nep) and FITC (for A $\beta$ ), followed by analysis with the LSCM as described previously (Rockenstein et al., 2001).

\section{Results}

Expression of active neprilysin from

Lenti-Nep-transduced cells

Human 293T cells were infected with a lentiviral vector expressing wild-type human neprilysin (Fig. 1a). The transduced cells were harvested and immunostained with an FITC-conjugated anti-neprilysin antibody, and subsequent flow cytometric analysis showed clear labeling for neprilysin on the cell surface (Fig. $1 b)$. The expression of neprilysin was also detected from transduced 293T and differentiated neural progenitor (HCN) cell lysates by immunoblot (Fig. 1c). Transduced cells were shown to express biologically active neprilysin by a colorimetric activity assay (see Materials and Methods). 293T cells infected with the

a

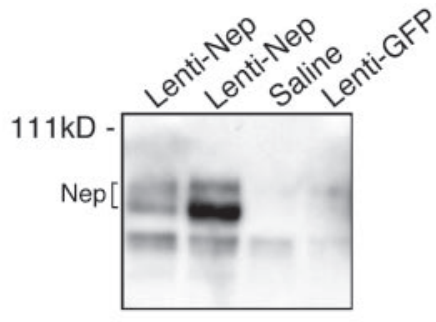

b

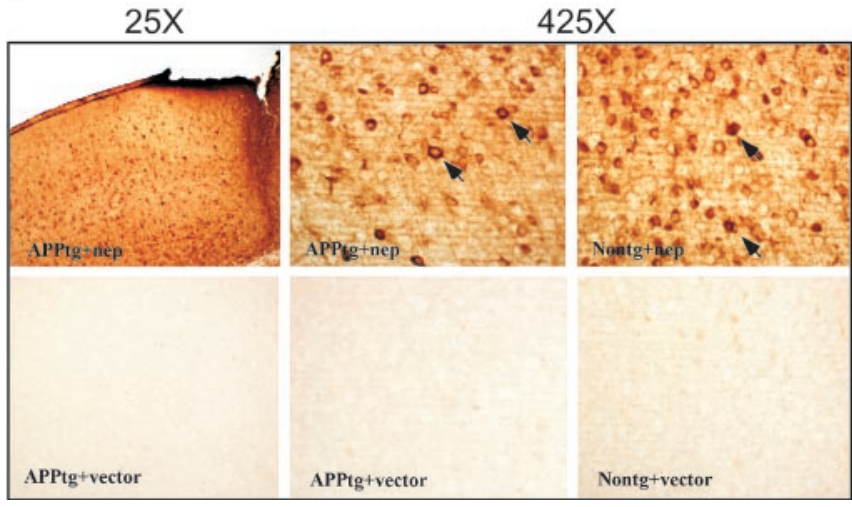

Figure 2. Expression of neprilysin in mouse brains. $a$, Detection of human neprilysin expression by immunoblot. Lenti-GFP and saline injections were used as negative controls. The quantity of total protein extracted from mouse brains was similar among all samples (data not shown). $b$, Detection of human neprilysin by immunohistochemistry (Lenti-Nep, top panels, arrows indicate examples of Nep-positive cells; Lenti-GFP, bottom panels, APPtg, J9M; Nontg, nontransgenic mouse; nep, Lenti-Nep injected; vector, Lenti-GFP injected).

Lenti-Nep vector [multiplicity of infection (m.o.i.) 50] produced an activity of $617 \mathrm{nmol} \cdot \mathrm{min}^{-1} \cdot \mathrm{mg}^{-1}$, whereas Lenti-GFP infected cells produced only $0.7 \mathrm{nmol} \cdot \mathrm{min}^{-1} \cdot \mathrm{mg}^{-1}$. Human kidney (highest neprilysin-expressing tissue) extract produced an activity of $24 \mathrm{nmol} \cdot \mathrm{min}^{-1} \cdot \mathrm{mg}^{-1}$. Furthermore, 293T cells infected with serial dilutions of the Lenti-Nep vector showed the ability to degrade synthetic $A \beta_{1-42}$ in a dose-dependent manner (Fig. 1d), as measured by ELISA. Differentiated neural progenitor cells (HCN) (Ray et al., 1995)

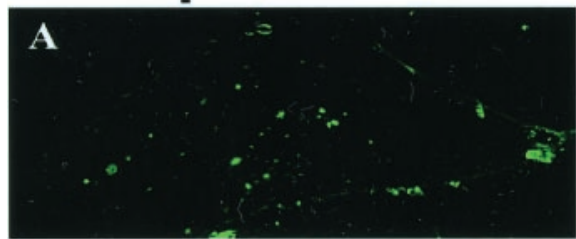

\section{Contralateral}
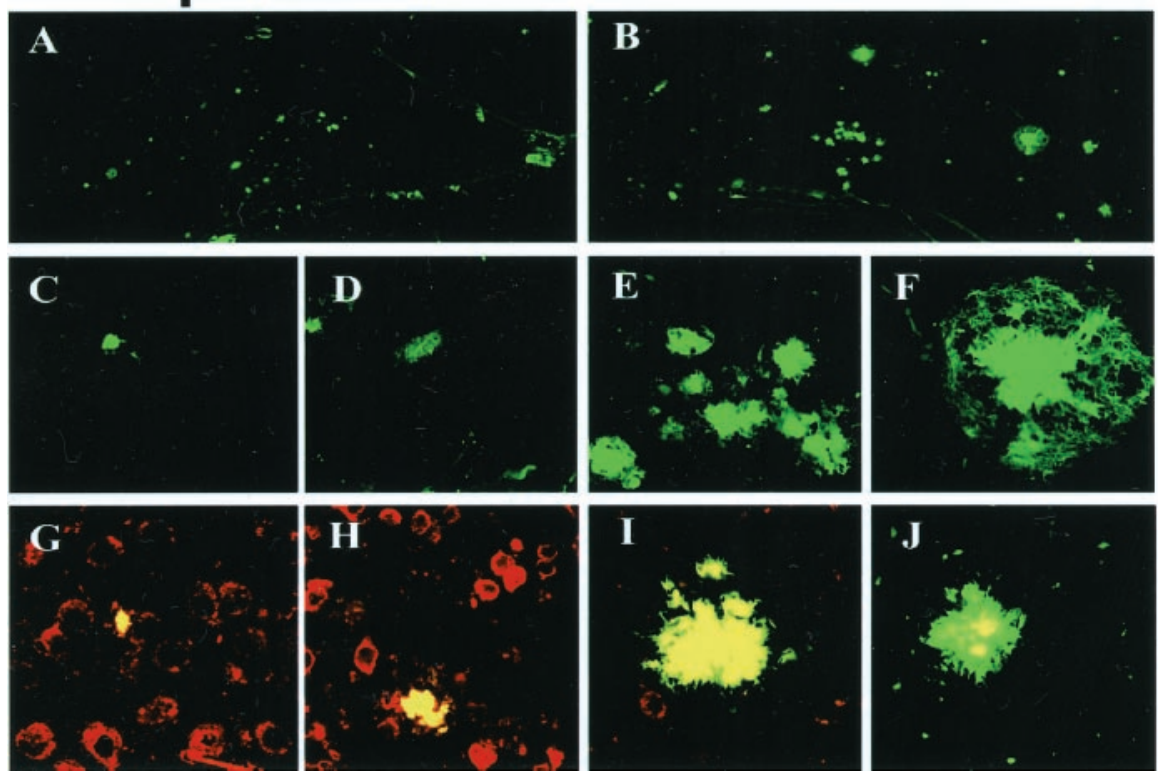

Figure 3. Reduced amyloid plaque formation in mice injected with Lenti-Nep. Shown are hippocampal sections from a representative transgenic-APP mouse (TASD41) injected with Lenti-Nep into the hippocampus and frontal cortex (right side injected: $A$, $(, D, G, H$; contralateral side: $B, E, F, I, J)$ (amyloid = green). Double immunolabeling for neprilysin and amyloid $(G-J)($ nep $=$ red $)$. infected with the Lenti-Nep vector (m.o.i. 50) also showed the ability to degrade both $\mathrm{A} \beta_{1-42}$ (Fig. $1 e$ ) and $\mathrm{A} \beta_{1-40}$ (data not shown). This activity was reduced by thiorphan, an inhibitor of neprilysin. Additionally, an inactive mutant of neprilysin (NepX) was unable to degrade $A \beta$ (Fig. $1 e)$. We conclude that Lenti-Nep can efficiently transduce cells in vitro and generate biologically active neprilysin.

\section{Neprilysin expression in the brain}

Transgenic (APP-tg) and nontransgenic mice were injected with $3 \mu$ l of Lenti-Nep vector into the hippocampus. One week later we removed the brains, and the injected hemispheres were homogenized in RIPA buffer. Neprilysin was detected in the brain homogenates by immunoblot (Fig. 2a). The expression of neprilysin was also detectable by immunohistochemistry on sections from injected mouse brains 1 
month after injection (Fig. 2b). Expression was typically localized to the injection site (covering a radius of $>200 \mu \mathrm{m}$ ); however, in some cases human neprilysin expression was detectable at more distant sites (data not shown). The distribution of Nep expression was similar to the distribution of GFP expression after the injection of a Lenti-GFP vector (data not shown).

\section{Amyloid- $\boldsymbol{\beta}$ deposits are reduced in Lenti-Nep-transduced brains}

To assess the potential anti-amyloidogenic effects of neprilysin in vivo, a lentiviral vector expressing this gene was injected into the CNS of APP transgenic mice from two independent lines that develop high levels of amyloid deposition. Amyloid plaques on the Lenti-Nep-injected hemispheres were qualitatively smaller compared with those on the contralateral side (Fig. 3, compare $A$, $C, D$ with $B, E, F)$. The use of double immunolabeling for neprilysin and amyloid showed that the appearance of small plaques colocalized with strong staining for neprilysin (Fig. 3, compare $G$, $H$ with $I, J)$. Amyloid quantification showed a significant decrease in the levels of amyloid deposition compared with the contralateral site ( $57 \pm 16 \%)$ (Fig. 4$)$. In contrast, control experiments in which transgenic mice were treated with Lenti-GFP, Lenti-NepX (mutant Nep), Lenti-iNep (heat-inactivated Nep vector), or saline alone showed comparable levels of amyloid deposition on both sides of the brain (100 $\pm 16.5 \%)$. Similar results were also obtained with Thioflavine-S staining (data not shown). Consistent with these findings, analysis of dendritic integrity with an antibody against MAP2 showed that treatment with Lenti-Nep was also associated with amelioration of the neurodegenerative process compared with the contralateral side, as shown by an average $16.2 \pm 7 \%$ increase in MAP2 immunoreactivity on the ipsilateral side. In contrast, control-treated mice showed similar levels of neurodegeneration between the two hemibrains, as shown by an average $3.7 \pm 11 \%$ decrease in MAP2 immunoreactivity on the ipsilateral side $(p<0.01)$.

\section{Discussion}

The present study shows that Lenti-Nep reduced AD-like pathology in transgenic mice. This finding is consistent with previous studies showing that Nep plays an important role in the degradation of $\mathrm{A} \beta$ in the brain. For example, downregulation of neprilysin genetically or with chemical inhibitors results in increased $\mathrm{A} \beta$ concentrations in the brain (Iwata et al., 2000, 2001), and gene transfer of Nep reduces $A \beta$ concentrations in vitro (Hama et al., 2001). Furthermore, it has been found that there is an inverse correlation between the presence of neprilysin and susceptibility to amyloid plaque formation in mice (Fukami et al., 2002). Similar findings have been reported in humans; decreased neprilysin mRNA levels were found to be associated with areas of high amyloid plaque burdens, colocalizing with increased APP and PS mRNA levels (Yasojima et al., 2001a). This inverse correlation is further supported by immunohistochemical localization of neprilysin in similar areas of the human brain (Akiyama et al., 2001; Yasojima et al., 2001b). Although it has been demonstrated that the infusion of Nep inhibitors can accelerate $A \beta$ deposition and that Nep induction is correlated with reduced $A \beta$ deposition (Iwata et al., 2000; Mohajeri et al., 2002), to our knowledge no direct effect on the accumulation of $\mathrm{A} \beta$ deposits had been established for Nep in vivo. Our study demonstrated that Nep does have an inhibitory effect on $\mathrm{A} \beta$ deposition in mice, further supporting a regulatory role for this endopeptidase in $\mathrm{AD}$. However, it is still unclear whether modulation of neprilysin expression or a

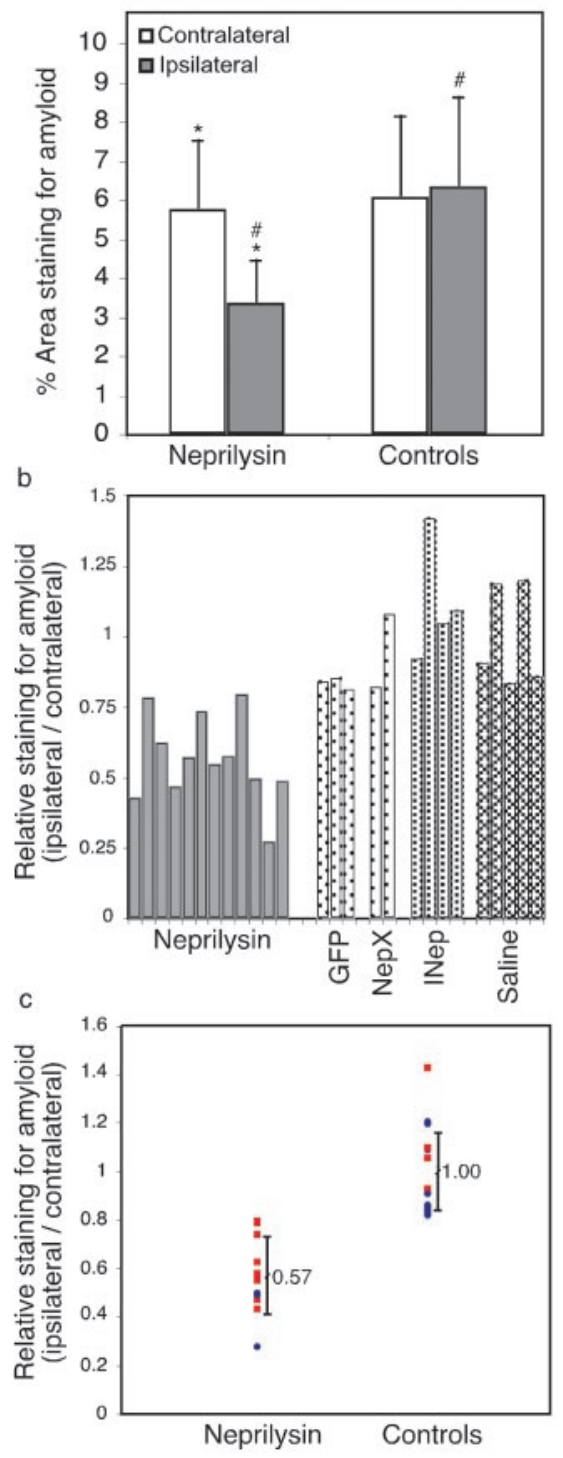

Figure 4. Comparison of plaque burdens in Lenti-Nep and control-treated transgenic mice. $a$, Mean absolute values for amyloid staining in Lenti-Nep (Neprilysin) and control-treated animals on the contralateral and ipsilateral hemispheres $\left({ }^{*} p=0.0007 ;{ }^{\#} p=0.06 ; t\right.$ test). Error bars represent SE. $b$, Relative ratios of ipsilateral (injected) versus contralateral (noninjected) plaque burdens. Nep, Lenti-Nep injected; GFP, Lenti-GFP injected; NepX, Lenti-NepX injected; INep, heat inactivated Lenti-Nep injected; Saline, saline injected. c, Scatter plot showing individual and the average relative values of the neprilysin $(n=12)$ and control $(n=14)$ groups; $p=0.000001$ ( $t$ test) (TASD41: red squares, $p=0.01$; J9M: blue circles, $p=0.004 ; t$ test). Error bars represent SD.

activity is intimately involved in the etiology of $\mathrm{AD}$, because genetic linkage analysis has shown no correlation between the neprilysin locus and risk for development of AD (Sodeyama et al., 2001; Oda et al., 2002).

The data presented for each mouse in Figure $4, b$ and $c$, were controlled internally by individually comparing the injected side with the contralateral side. This method of analysis compensated for the variability in total plaque load seen between mice (Fig. 4a) and demonstrated more clearly the localized effects of neprilysin expression. The localization of these effects to the injected hemisphere was expected, because wild-type neprilysin is a type II integral membrane protein. Therefore, its effects were likely mediated through localized depletion of $\mathrm{A} \beta$ near the sites of expres- 
sion. The mechanisms through which Nep might reduce amyloid deposition include either increased degradation or reduced growth of already existing plaques. Our studies support the possibility that both mechanisms are at work, because plaques found in regions strongly expressing neprilysin were observed to be smaller and more compact, and in some cases, plaque load was reduced to $<50 \%$ of that found on the contralateral side (Figs. 3 , 4). Considering the short time period involved (1 month), it is unlikely that the greater load on the contralateral side was solely the result of more rapid growth. Therefore, at least in these more dramatic cases, it would seem that plaques on the Lenti-Nepinjected sides were reduced.

Plaque assembly involves several stages that include $A \beta$ oligomerization and profibral formation that might serve as the nucleating nidus for $\mathrm{A} \beta$ polymerization and fibril formation (Zerovnik, 2002). The role of $A \beta$ oligomers and fibrils in neurotoxicity in $\mathrm{AD}$ is still highly controversial; however, some studies support a role for oligomers in neurotoxicity. It has been suggested recently that oligomers of $\mathrm{A} \beta$ are the major form interfering with long-term potentiation in mice (Walsh et al., 2002). The overexpression of neprilysin would likely reduce the local concentration of $A \beta$ oligomers through the degradation of monomers and possibly oligomers and fibrils directly. However, the potential dissociation of fibrils could also increase the local oligomer concentration and thus be harmful. Although our study did not directly address this issue, it did demonstrate that neprilysin affects the $A \beta$ dynamics, such that amyloid plaques either develop more slowly or dissociate. Furthermore, this effect was associated with reduced neurodegeneration, as revealed by MAP2 immunocytochemistry. This apparent reduction in neurodegeneration could be the result of protection of neurons from toxic $A \beta$ products, enhanced regeneration, or simply upregulation of MAP2 expression. Regardless, this finding implies that Nep gene transfer did not generate harmful products but rather had a beneficial effect in vivo. In conclusion, our study supports a role for neprilysin in the regulation of amyloid deposition and highlights the potential use of gene therapy approaches for treatment of Alzheimer's disease.

\section{References}

Akiyama H, Kondo H, Ikeda K, Kato M, McGeer PL (2001) Immunohistochemical localization of neprilysin in the human cerebral cortex: inverse association with vulnerability to amyloid beta-protein (Abeta) deposition. Brain Res 902:277-281.

Dull T, Zufferey R, Kelly M, Mandel RJ, Nguyen M, Trono D, Naldini L (1998) A third-generation lentivirus vector with a conditional packaging system. J Virol 72:8463-8471.

Follenzi A, Ailles LE, Bakovic S, Geuna M, Naldini L (2000) Gene transfer by lentiviral vectors is limited by nuclear translocation and rescued by HIV-1 pol sequences. Nat Genet 25:217-222.

Fukami S, Watanabe K, Iwata N, Haraoka J, Lu B, Gerard NP, Gerard C, Fraser P, Westaway D, George-Hyslop PS, Saido TC (2002) Abetadegrading endopeptidase, neprilysin, in mouse brain: synaptic and axonal localization inversely correlating with Abeta pathology. Neurosci Res 43:39-56.

Glabe C (2000) Does Alzheimer disease tilt the scales of amyloid degradation versus accumulation? Nat Med 6:133-134.

Hama E, Shirotani K, Masumoto H, Sekine-Aizawa Y, Aizawa H, Saido TC
(2001) Clearance of extracellular and cell-associated amyloid beta peptide through viral expression of neprilysin in primary neurons. J Biochem (Tokyo) 130:721-726.

Iwata N, Tsubuki S, Takaki Y, Watanabe K, Sekiguchi M, Hosoki E, Kawashima-Morishima M, Lee HJ, Hama E, Sekine-Aizawa Y, Saido TC (2000) Identification of the major Abeta1-42-degrading catabolic pathway in brain parenchyma: suppression leads to biochemical and pathological deposition. Nat Med 6:143-150.

Iwata N, Tsubuki S, Takaki Y, Shirotani K, Lu B, Gerard NP, Gerard C, Hama E, Lee HJ, Saido TC (2001) Metabolic regulation of brain Abeta by neprilysin. Science 292:1550-1552.

Li C, Hersh LB (1995) Neprilysin: assay methods, purification, and characterization. Methods Enzymol 248:253-263.

Masliah E, Sisk A, Mallory M, Mucke L, Schenk D, Games D (1996) Comparison of neurodegenerative pathology in transgenic mice overexpressing V717F $\beta$-amyloid precursor protein and Alzheimer's disease. J Neurosci 16:5795-5811.

Miyoshi H, Blomer U, Takahashi M, Gage FH, Verma IM (1998) Development of a self-inactivating lentivirus vector. J Virol 72:8150-8157.

Mohajeri MH, Wollmer MA, Nitsch RM (2002) $\mathrm{A} \beta_{42}$-induced increase in neprilysin is associated with prevention of amyloid plaque formation in vivo. J Biol Chem 277:35460-35465.

Mucke L, Masliah E, Yu GQ, Mallory M, Rockenstein EM, Tatsuno G, Hu K, Kholodenko D, Johnson-Wood K, McConlogue L (2000) High-level neuronal expression of $\mathrm{A} \beta 1-42$ in wild-type human amyloid protein precursor transgenic mice: synaptotoxicity without plaque formation. J Neurosci 20:4050-4058.

Naldini L, Blomer U, Gage F, Trono D, Verma I (1996) Efficient transfer, integration, and sustained long-term expression of the transgene in adult rat brains injected with a lentiviral vector. Proc Natl Acad Sci USA 93:11382-11388.

Oda M, Morino H, Maruyama H, Terasawa H, Izumi Y, Torii T, Sasaki K, Nakamura S, Kawakami H (2002) Dinucleotide repeat polymorphisms in the neprilysin gene are not associated with sporadic Alzheimer's disease. Neurosci Lett 320:105-107.

Ray J, Raymon HK, Gage FH (1995) Generation and culturing of precursor cells and neuroblasts from embryonic and adult central nervous system. Methods Enzymol 254:20-37.

Reilly CE (2001) Neprilysin content is reduced in Alzheimer brain areas. J Neurol 248:159-160.

Rockenstein E, Mallory M, Mante M, Sisk A, MasliahaE (2001) Early formation of mature amyloid-beta protein deposits in a mutant APP transgenic model depends on levels of Abeta(1-42). J Neurosci Res 66:573-582.

Sodeyama N, Mizusawa H, Yamada M, Itoh Y, Otomo E, Matsushita M (2001) Lack of association of neprilysin polymorphism with Alzheimer's disease and Alzheimer's disease-type neuropathological changes. J Neurol Neurosurg Psychiatry 71:817-818.

Turner AJ, Isaac RE, Coates D (2001) The neprilysin (NEP) family of zinc metalloendopeptidases: genomics and function. BioEssays 23:261-269.

Walsh DM, Klyubin I, Fadeeva JV, Cullen WK, Anwyl R, Wolfe MS, Rowan MJ, Selkoe DJ (2002) Naturally secreted oligomers of amyloid beta protein potently inhibit hippocampal long-term potentiation in vivo. Nature 416:535-539.

Yasojima K, McGeer EG, McGeer PL (2001a) Relationship between beta amyloid peptide generating molecules and neprilysin in Alzheimer disease and normal brain. Brain Res 919:115-121.

Yasojima K, Akiyama H, McGeer EG, McGeer PL (2001b) Reduced neprilysin in high plaque areas of Alzheimer brain: a possible relationship to deficient degradation of beta-amyloid peptide. Neurosci Lett 297:97-100.

Zerovnik E (2002) Amyloid-fibril formation. Eur J Biochem 269:3362-3371.

Zufferey R, Donello JE, Trono D, Hope TJ (1999) Woodchuck hepatitis virus posttranscriptional regulatory element enhances expression of transgenes delivered by retroviral vectors. J Virol 73:2886-2892. 